
a) CHIRPS
b) Multimodel mean
c) Biais
d) Taylor diagram
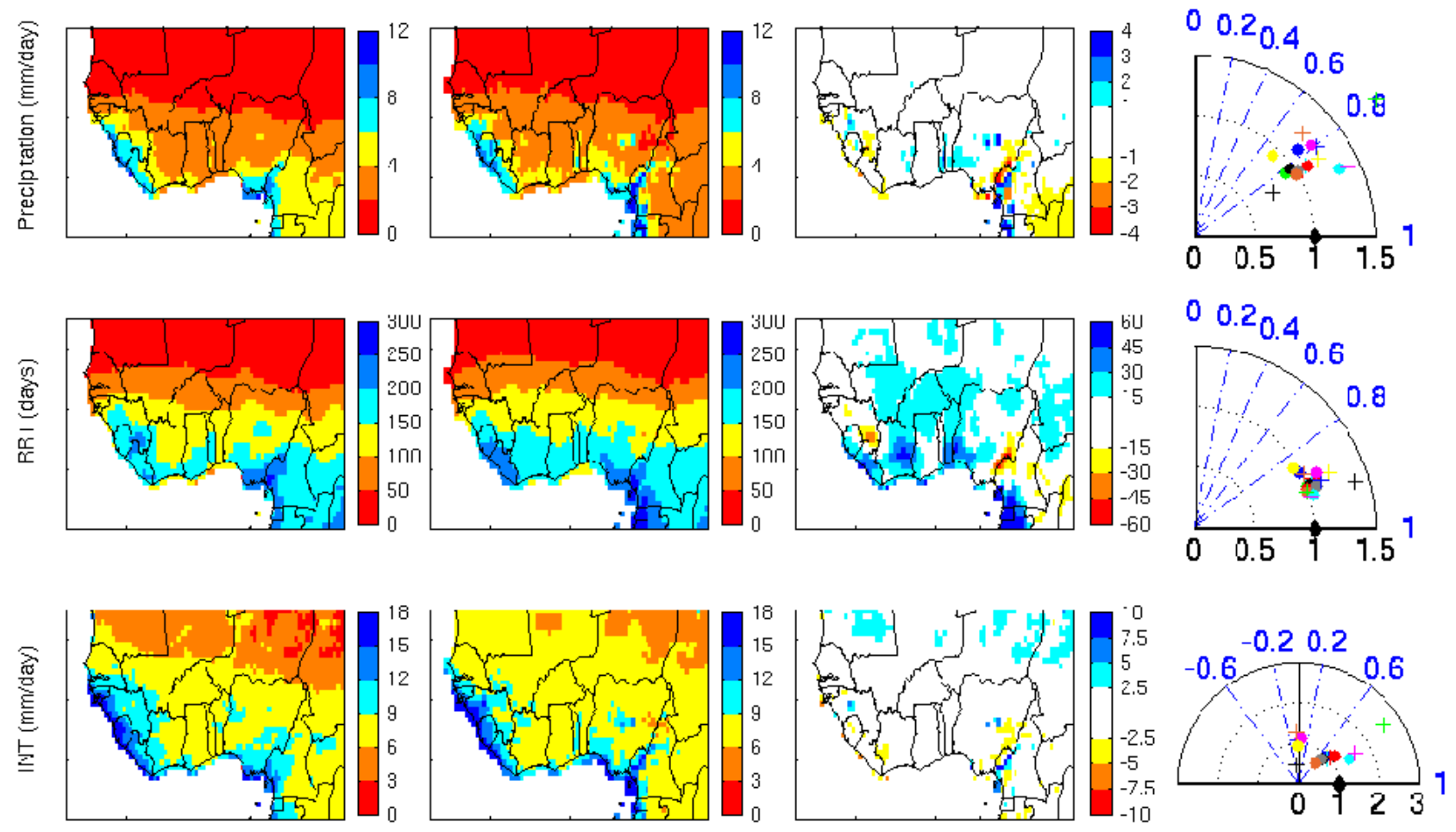

Supplementary Figure 1. Annual precipitation (top raw, mm/day), RR1 (middle row, days), and INT (bottom raw, mm/day) averaged over 1981-2014, and shown as a) observed in the CHIRPS dataset, b) simulated by the CORDEX multimodel mean, c) ensemble mean bias (relative to the observations), and d) Taylor diagram (Taylor 2001). In the Taylor diagram, the CHIRPS observations are represented by the black diamond, and individual CORDEX simulations are represented by different colors following the color code of Table 1. 
A/ Dakar

a) Daily mean precipitation

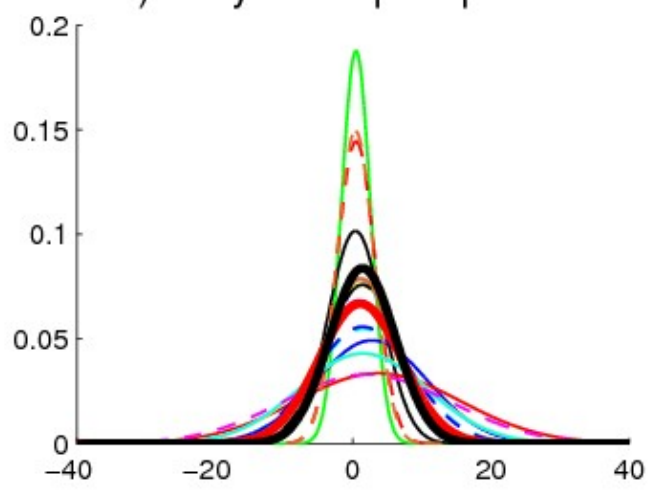

c) RR1

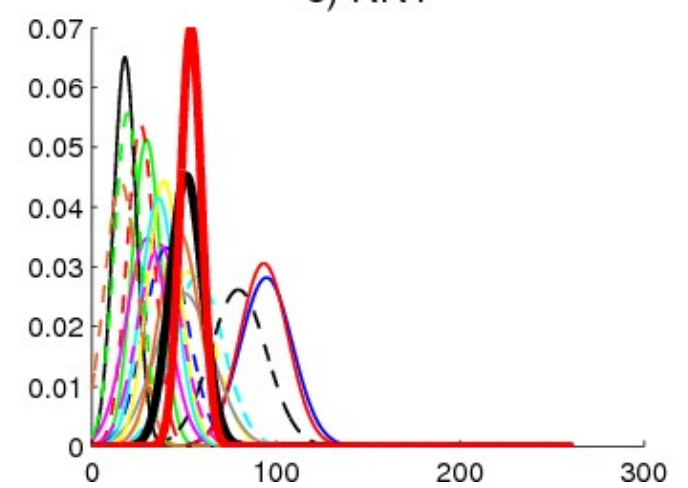

B/ Ouagadougou

a) Daily mean precipitation

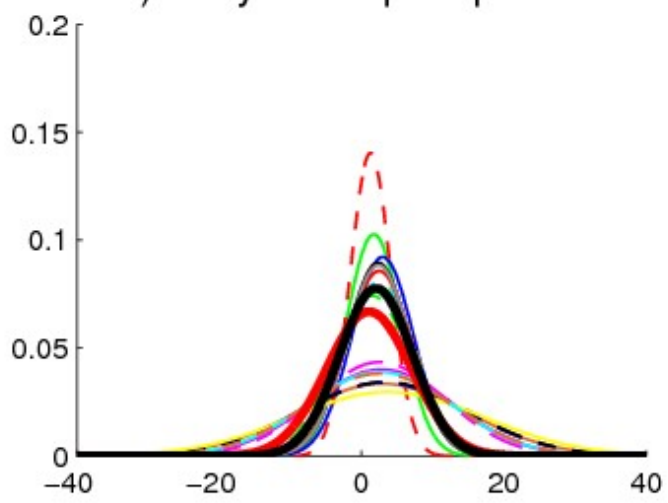

c) RR1

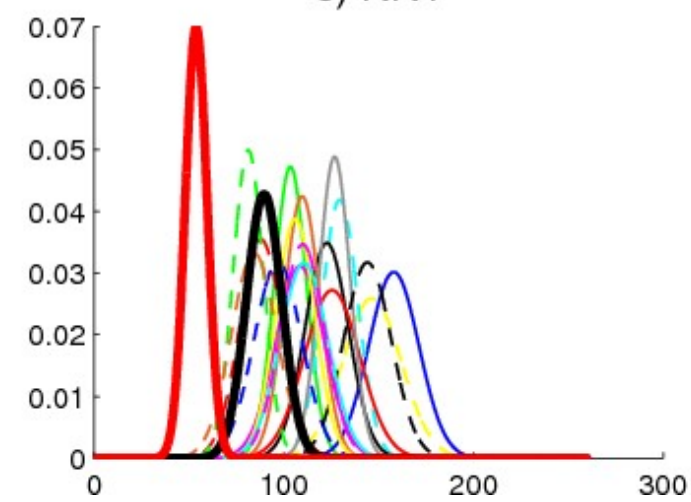

b) Yearly mean precipitation

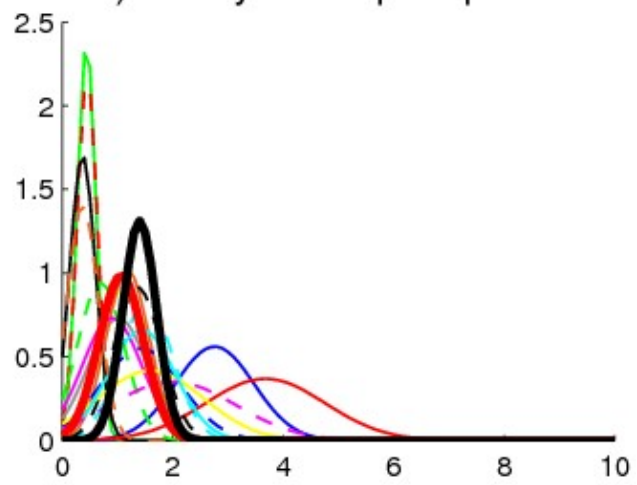

d) INT

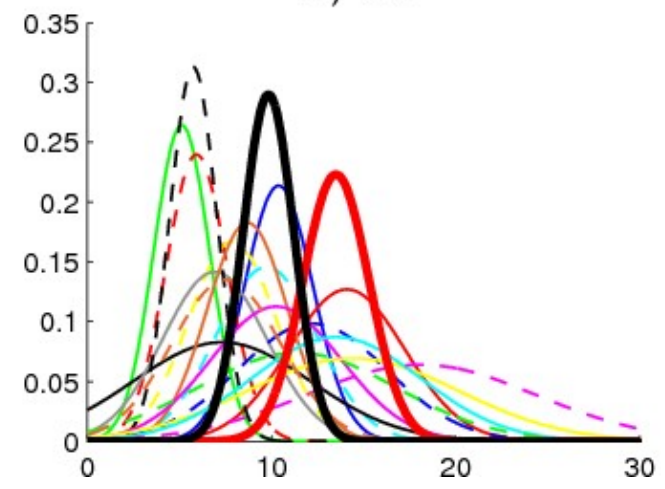

b) Yearly mean precipitation

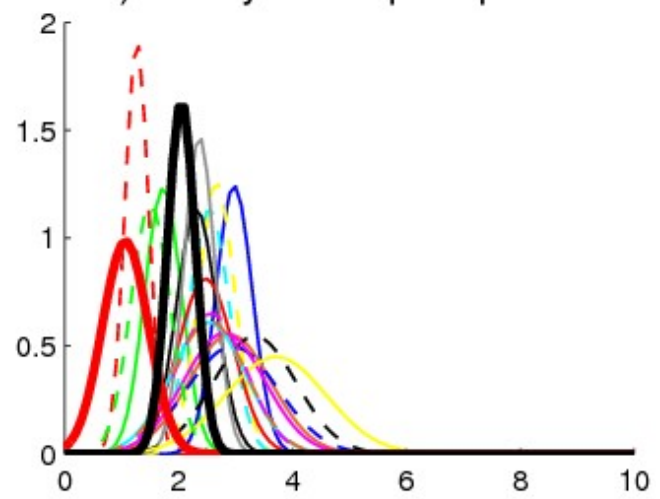

d) INT

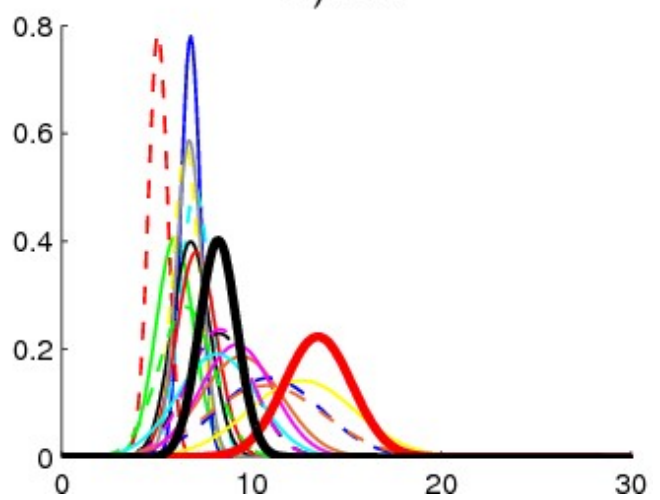




\section{C/ Accra}

a) Daily mean precipitation

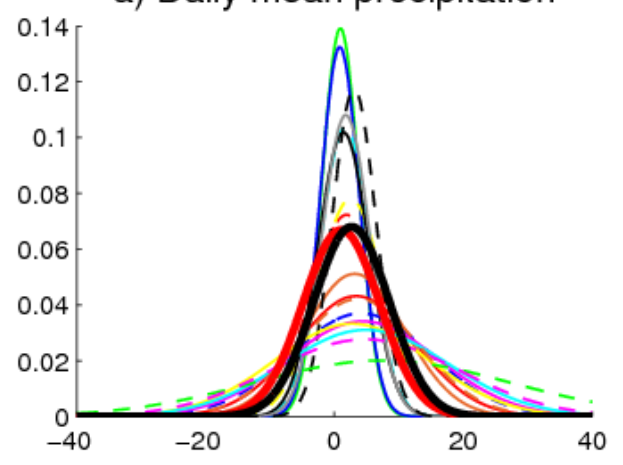

c) RR1

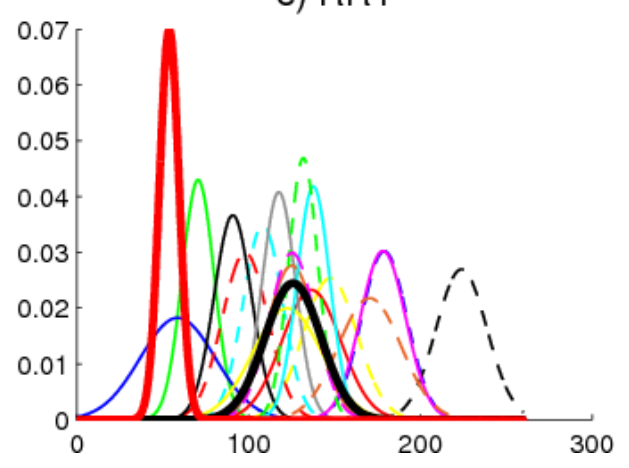

b) Yearly mean precipitation

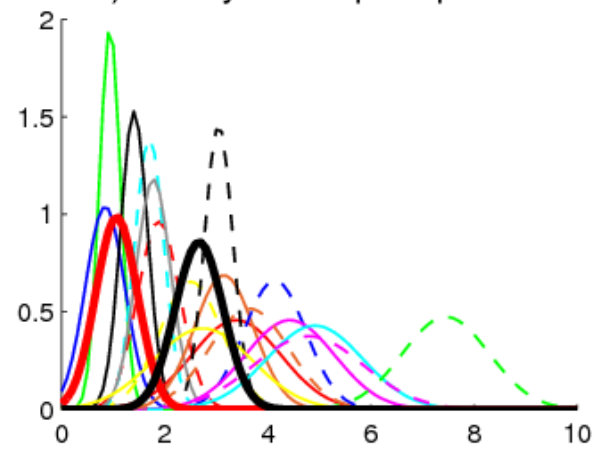

d) INT

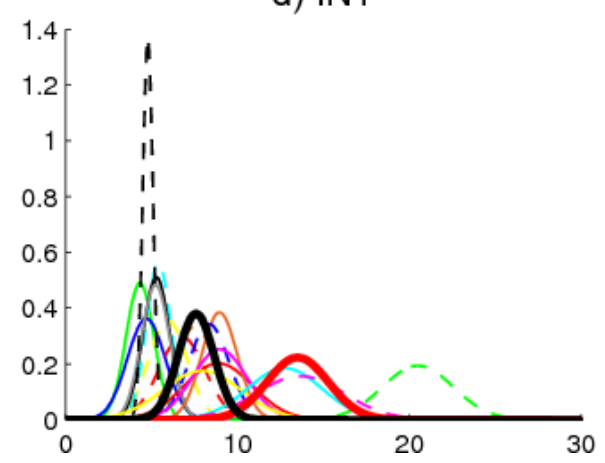

Supplementary Figure 2. Statistical distribution of a) daily mean precipitation, b) yearly mean precipitation, c) RR1, and d) INT, as observed in the BADOPLUS dataset (thick red curve), the CHIRPS dataset (thick black curve), and as simulated in the individual CORDEX simulations (colored curves following the color code of Table 1) over 1981-2014. Shown are the results for A/ Dakar, B/ Ouagadougou, and C/ Accra. 